\title{
Predicción del Precio de la Electricidad en la Bolsa mediante un Modelo Neuronal No-Lineal Autorregresivo con Entradas Exógenas
}

\author{
Adriana P. Agudelo, Jesús M. López-Lezama y Esteban Velilla \\ Grupo de Investigación GIMEL, Departamento de Ingeniería Eléctrica, Facultad de Ingeniería, \\ Universidad de Antioquia, Calle 70 No.52-21, Medellín - Colombia (e-mail: adrianagudelom@gmail.com; \\ jmaria.lopez@udea.edu.co; esteban.velilla@udea.edu.co).
}

Recibido May. 20, 2015; Aceptado Ago. 5, 2015; Versión final Ago. 25, 2015, Publicado Dic. 2015

\begin{abstract}
Resumen
Se realizó la predicción del precio de energía de bolsa promedio mensual en el mercado eléctrico colombiano por medio de un modelo de redes neuronales no-lineal autorregresivo. Considerando como entradas exógenas: la demanda, la relación entre generación hidráulica-térmica, la probabilidad del Fenómeno de El Niño y el volumen útil diario energía; y como variable autorregresiva el precio de bolsa promedio mensual. Para el entrenamiento, validación y pruebas se analizaron datos entre enero de 2003 y marzo de 2014. Adicionalmente, la efectividad del modelo fue probada con datos entre abril de 2014 y febrero de 2015. Se observó que al incorporar la historia del precio y variables dependientes de condiciones hidrológicas como el Fenómeno del Niño en el modelo, se puede reproducir adecuadamente la dinámica del precio de la energía en Colombia, incluso en meses posteriores al periodo de tiempo considerado.
\end{abstract} de tiempo

\section{Forecasting Electricity Stock Price by Means of a Nonlinear Autoregressive Neural Model with Exogenous Inputs}

\begin{abstract}
The forecasting of the monthly average stock price of Colombian electricity was carried out by means of a nonlinear autoregressive exogenous model. Such a model considers as exogenous inputs the demand, the ratio between hydraulic and thermal generation, the probability of El Niño phenomenon and the daily available energy volume; and as an autoregressive variable the monthly average stock price. For training, validation and testing, data corresponding to the period January 2003 to March 2014 were considered. Furthermore, the effectiveness of the model was tested with data for the period April 2014 to February 2015. This shows that the incorporation of historic data of prices and variables that depend on hydrologic conditions, such as El Niño phenomenon, allows reproducing the dynamics of Colombia's energy prices, even for forthcoming periods.
\end{abstract}

Keywords: spot price, hydrology, artificial neural networks, autoregressive models, time series 


\section{INTRODUCCIÓN}

Uno de los aspectos más importantes en la desregulación de los mercados eléctricos es la apertura a la participación de inversionistas privados, con el consecuente incremento de la competitividad en el sector, lo que se puede ver reflejado en los precios. El precio de la energía en los mercados desregulados puede ser altamente volátil, particularmente en aquellos que presentan una alta componente hidrológica. Otros factores que influyen en el precio, aparte de la hidrología, son las restricciones eléctricas del sistema, el comportamiento estratégico de los agentes, la expectativa de precios de combustibles, etc (UPME, 2013).

La predicción del precio de bolsa de energía ha sido abordada mediante diversos modelos y metodologías, siendo los más referenciados los modelos de predicción de series de tiempo, modelos ARIMA, GARCH, redes neuronales, método de Montecarlo y métodos híbridos (Botero et al., 2007). En particular, en el caso de las redes neuronales, éstas permiten establecer relaciones no lineales entre variables de entrada y salida, facilitando que su paradigma computacional sea aplicable en diversos campos del conocimiento (Agudelo et al., 2014), (Velilla et al., 2014).

Areekul et al. (2010) ofrecen una metodología híbrida que combina el modelo ARIMA con redes neuronales artificiales (ANN) para la predicción de precios de electricidad a corto plazo. Los resultados indicaron que un modelo híbrido ARIMA-ANN puede mejorar la precisión de las previsiones de precio. Conejo et al. (2005) utilizan la Transformada de Waveley y el modelo ARIMA para la predicción de precios en el corto plazo. En este caso la serie histórica de precios se descompone usando la transformada de Wavelet, luego se pronostican los valores de la serie utilizando el modelo ARIMA. Las predicciones con ARIMA permiten, a través de la transformada inversa de Wavelet, reconstruir el comportamiento de las series de los precios.

En el modelo de red neuronal difusa propuesto por Amjady (2006), la red predice precios horarios con un día de antelación, mediante una combinación de lógica difusa y un algoritmo de aprendizaje. Anbazhagan \& Kumarappan (2013) presentan un modelo de red neuronal recurrente para la predicción a corto plazo usando el modelo de red de Elman. Según los autores, este modelo puede obtener resultados similares a otros modelos de predicción como el modelo ARIMA y redes neurodifusas en menor tiempo computacional. Catalão et al. (2011) presentan un enfoque híbrido que utiliza la transformada de Wavelet, redes neuronales y lógica difusa para la predicción de precios en el corto plazo en un mercado eléctrico competitivo. García et al. (2005) describen un modelo generalizado condicional autoregresivo heterocedástico (GARCH) que permite analizar datos de series de tiempo en general, el cual es aplicado a la predicción de precios en el mercado español y californiano. Otros autores como Botero y Cano (2008) han realizado predicción de precios por medio de series de tiempo con modelos de regresión con base en series históricas de precios. Weron y Misiorek (2005) estudian modelos con series de tiempo ARMA y ARMAX con entradas exógenas. También se han utilizado modelos con redes neuronales autorregresivas en el mercado eléctrico brasilero como el reportado en (Velásquez et al., 2008). Nogales et al. (2002) realizan una regresión dinámica para predicción de precios. En este caso los precios de una hora están relacionados con los valores pasados del precio y se utiliza un modelo de función de transferencia donde se asume que el precio y la demanda son estacionarios. Villada et al. (2011) realizan la predicción de precios de energía eléctrica en Colombia mediante el uso de redes neurodifusas. En este caso se utilizaron dos estructuras de red; una tomó como entrada la serie de precios diarios y la segunda, la serie de precios diarios y el nivel medio de los embalses, obteniéndose mejores resultados en el pronóstico con la última alternativa. Barrientos et al. (2012) predicen el precio de la energía en Colombia a largo plazo con modelos de redes neuronales, considerando como variables de entrada la demanda y oferta de la electricidad y el nivel de los embalses.

Colombia presenta una alta dependencia de la generación hidráulica, debido a que aproximadamente $65 \%$ de la producción de energía es hidráulica. De igual manera, varios Fenómenos de El Niño ocurridos en Colombia han reflejado el impacto que tiene éste en el mercado por su alta dependencia de la hidrología. Esta situación se ha evidenciado en el aumento del precio de la electricidad cuando se anuncia el Fenómeno de EI Niño (CIDET, 2014). Lo cual sucede debido a que los generadores se deben preparar para enfrentar una época de fuerte sequía y deben tener especial cuidado con los niveles de los embalses para no presentar racionamientos. El Fenómeno de El Niño incide directamente sobre los aportes hídricos a los embalses, conllevando a la disminución de la generación hidráulica y por ende, al aumento de la generación térmica, incrementando los precios de la energía en bolsa y en contratos a largo plazo (UPME, 2013).

En este artículo se realizó un pronóstico del precio de bolsa promedio mensual, en el mercado eléctrico colombiano, mediante un modelo de red neuronal no lineal autorregresivo con 4 entradas exógenas: la demanda, la probabilidad del Fenómeno de El Niño, la relación entre generación hidráulica y generación térmica, y el volumen útil diario de energía. Estas tres últimas variables tienen relación directa con las condiciones hidráulicas. Finalmente, el promedio del precio de bolsa mensual es utilizado como realimentación del modelo, considerándose así, la historia de éste. 
Debido a que el precio de bolsa de la energía eléctrica en Colombia es de naturaleza no lineal y puede ser representado como una serie de tiempo, se aprovecha el modelo NARX que combina redes neuronales, series de tiempo y no linealidad entre las variables de entrada con respecto a la de salida, para la predicción del precio promedio de bolsa.

\section{METODOLOGÍA}

En trabajos previos relacionados con la predicción del precio de la energía eléctrica en Colombia, se ha observado que éste presenta una alta dependencia de la hidrología (Barrientos et al., 2012). Razón por la cual en este artículo se optó por considerar 3 variables exógenas que tiene relación con la hidrología: la relación entre generación hidráulica y térmica, variable que indica el tipo de energía generada en mayor cantidad, cuando se presenta mayor generación térmica se dan mayores costos; el volumen útil diario de energía, variable que refleja la cantidad de energía hidráulica disponible, cuando este volumen disminuye aumenta el precio de la energía; y la probabilidad del fenómeno de El Niño, variable que incide directamente sobre los aportes hídricos a los embalses, conllevando a la disminución de la generación hidráulica y por ende, al aumento de la generación térmica, incrementando los precios de la energía en bolsa (UPME, 2013).

Adicionalmente, debido a que el precio resulta ser altamente volátil y exhibe un comportamiento no lineal, se optó por abordar su predicción con redes neuronales que pudieran incorporar no solo el comportamiento no lineal sino también tener en consideración los comportamientos dependientes de la historia de sus variables.

\section{Variables consideradas}

Toda la información, excepto la probabilidad del Fenómeno de El Niño, fue obtenida de la página de XM (Compañía de Expertos en Mercados S.A. E.S.P), el pronóstico del Fenómeno de El Niño fue adquirido de la página de IRI (International Research Institute for Climate and Society). Los datos fueron tomados desde enero de 2003 hasta marzo de 2014, lo que corresponde a 135 muestras. No se utilizó información desde la creación del mercado en 1994, ya que en Colombia se implementó un cambio regulatorio en 2001 que modificó la forma en que los agentes presentaban sus ofertas de precios (Resolución CREG 026/01). De igual manera, la información del pronóstico del Fenómeno de El Niño se encuentra desde marzo de 2002, por lo que se optó por trabajar con información desde enero de 2003. Con respecto a los precios, estos se llevaron a valor presente neto de marzo de 2014.Por otro lado, si bien no se considera una variable explícita que incluya los efectos de la regulación en el lapso de estudio, este efecto se ve reflejado en los datos históricos del precio, el cual reacciona a los cambios regulatorios.

El comportamiento entre la generación hidráulica y generación térmica en el periodo de tiempo analizado es ilustrado en la

Fig. 1, donde se aprecia que la generación eléctrica en Colombia es predominantemente hidráulica. En la Fig. 2se presenta el precio de bolsa promedio mensual llevado al valor presente neto de marzo de 2014 y la probabilidad de ocurrencia del Fenómeno de El Niño, en esta se observa que cuando la probabilidad de que se presente el Fenómeno de El Niño es alta el precio tiende a subir y cuando la probabilidad es baja el precio tiende a estabilizarse.

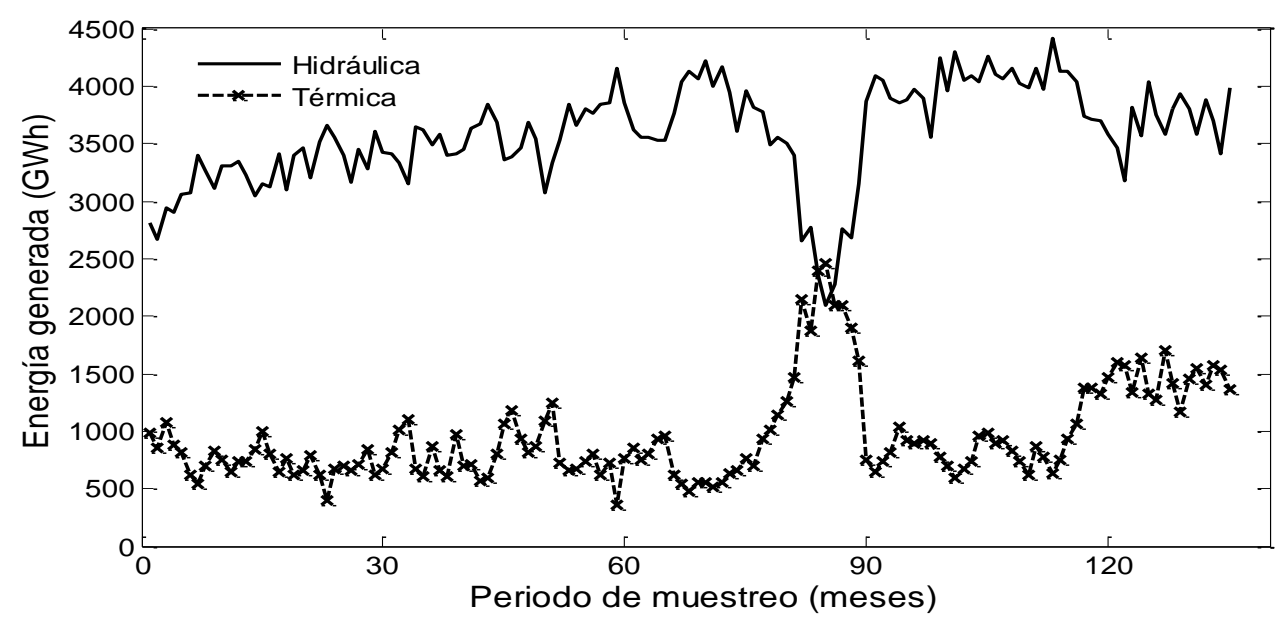

Fig. 1: Comportamiento de la generación hidráulica y térmica en Colombia 


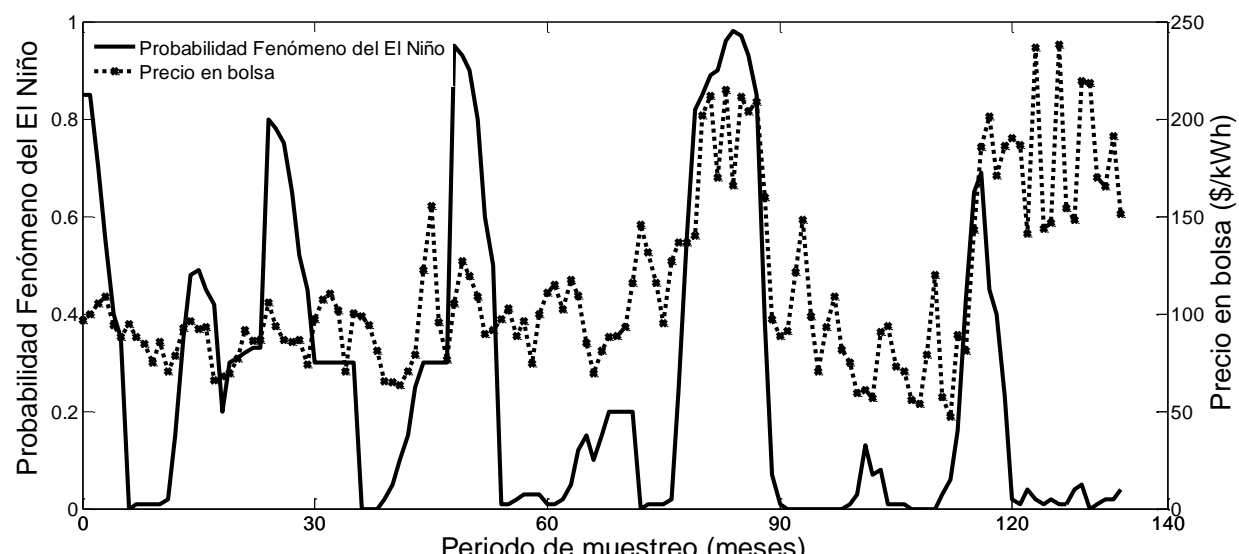

Fig. 2: Probabilidad del Fenómeno de El Niño y precio de bolsa promedio mensual

En la Fig. 3 se observa que cuando la relación entre generación hidráulica y generación térmica aumenta (la generación hidráulica es mayor que la generación térmica) el precio de bolsa es estable y cuando la relación disminuye (la generación hidráulica es menor que la generación térmica), el precio de bolsa aumenta. En cuanto al volumen útil diario de energía, cuando hay mayor volumen el precio de bolsa tiende a disminuir y viceversa, como se ilustra en Fig. 4

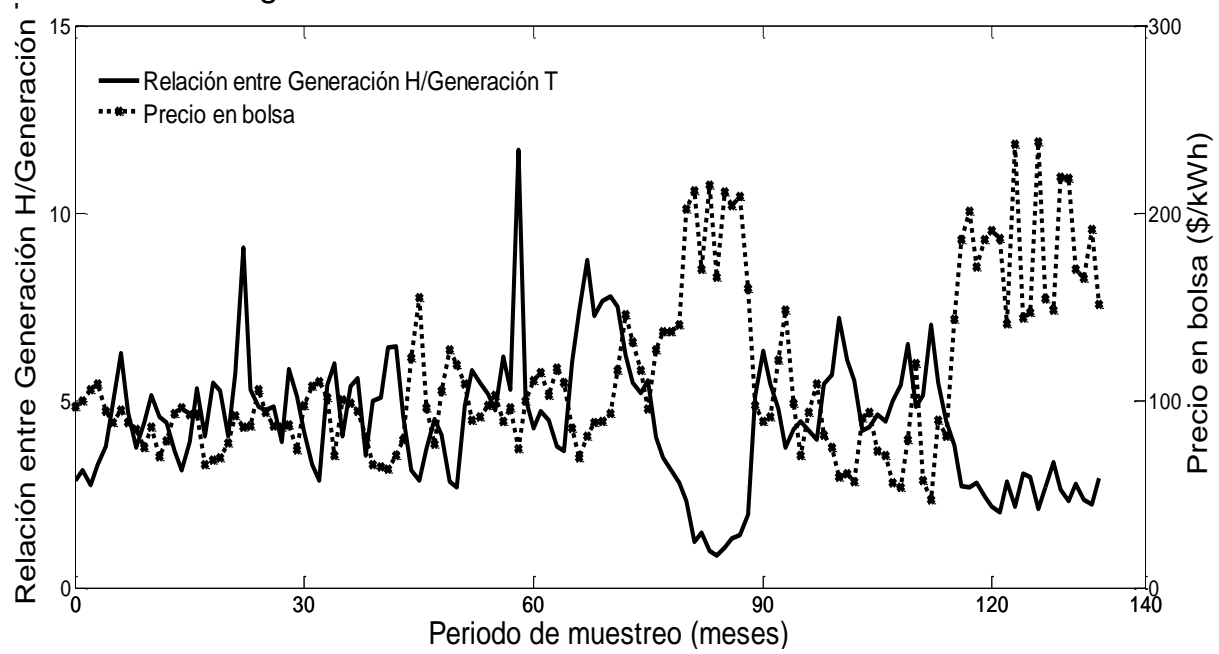

Fig. 3: Relación entre Generación Hidráulica/Generación Térmica y precio de bolsa promedio mensual

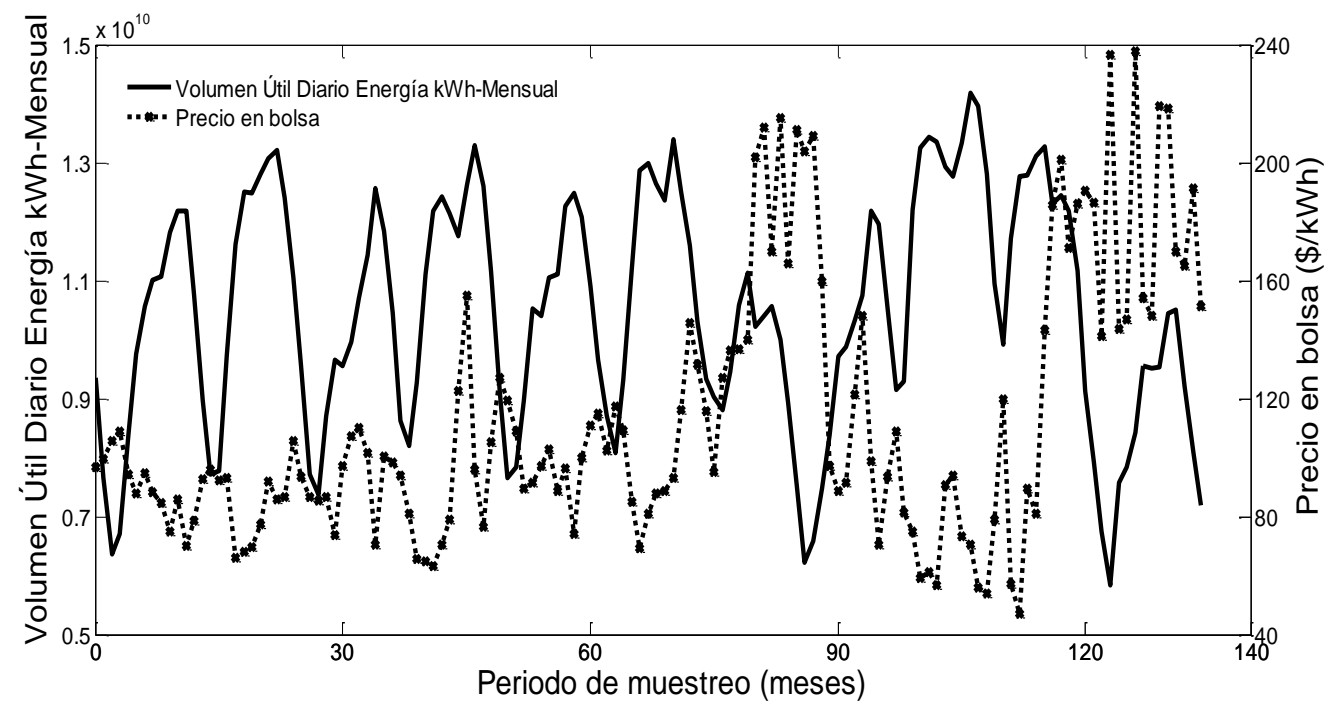

Fig. 4: Volumen útil diario de energía y precio de bolsa promedio mensual

Por lo anterior las entradas exógenas que se tuvieron en cuenta para el desarrollo de este artículo fueron las que involucraban condiciones hidrológicas como la probabilidad del Fenómeno de El Niño, el volumen útil diario de energía y la relación entre generación hidráulica y térmica. Además se consideró la demanda de energía ya que esta variable es fundamental en los estudios de predicción de precios de energía (Barrientos 
et al, 2012). Con el fin de observar la independencia de estas variables, se realizó el análisis de valores singulares. Para garantizar la independencia de las variables se requiere que todos los valores singulares sean diferentes de cero. Los valores singulares obtenidos en este análisis fueron de 14.11, 3.70, 1.65 y 1.02, indicando esto que todos son diferentes de cero y por tanto las entradas escogidas son linealmente independientes.

\section{Estructura de la red NARX}

El modelo implementado para realizar el pronóstico del precio de bolsa promedio mensual en el corto plazo es una red neuronal autorregresiva con entradas exógenas (NARX), como se ilustra en Fig. 5; la cual, es una red dinámica recurrente. Las redes recurrentes poseen conexiones que alimentan los estados de salida hacia la entrada del sistema, con lo cual, la red neuronal se convierte en un sistema no lineal realimentado (De la Fuente y Calonge, 2000). Los modelos autorregresivos se basan en que el valor actual de una serie y(t) puede explicarse en función de valores pasados de la misma variable.

Este modelo se caracteriza por tener la formulación expresada en (1), donde y(t) es la salida que en el caso de estudio sería el precio de bolsa promedio mensual de la energía eléctrica, $d$ es el número de retardos y $x(t)$ es la entrada exógena.

$$
y(t)=f(x(t-1), \ldots, x(t-d), y(t-1), \ldots, y(t-d))
$$

La estructura de la red neuronal NARX seleccionada posee 2 capas: la capa oculta que tiene contacto con las entradas de la red y la capa de salida relacionada con la salida del modelo. Ambas capas están conformadas por neuronas, las cuales se caracterizan por la función de activación o función de transferencia encargada de mapear los datos de entrada de la neurona con la finalidad de dar el estímulo de ésta o salida de la red. La función de activación de la primera capa fue la Tansig, y su expresión asociada está dada por (2), en la cual $n$ es el valor numérico de la entrada de la neurona. La función de activación de la capa de salida es la Purelin o lineal $(a=n)$, donde la entrada es igual a la salida, esta función de activación permite que la salida de la red pueda tomar cualquier valor.

$$
\mathrm{a}=\frac{1}{1+\mathrm{e}^{-2 \mathrm{n}}}
$$

La estructura de esta red es representada en la Fig. 5, donde X1...Xn son las entradas de la red, W son los pesos y $b$ las bias que se determinan a través del entrenamiento de la red.

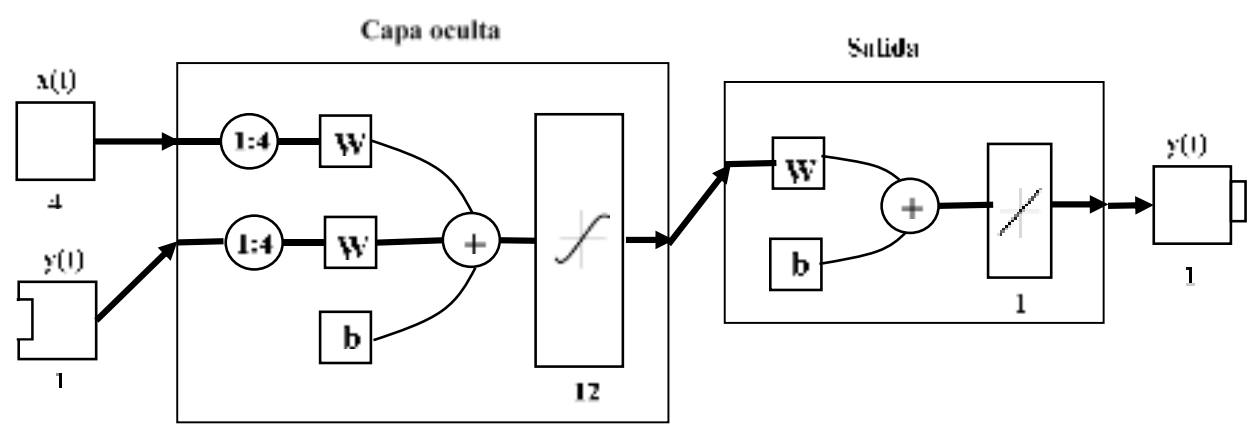

Fig. 5: Estructura de la red neuronal

Con la finalidad de completar el modelo NARX se hace necesario ajustar los parámetros de la estructura definida, esto es, los pesos (W) y las bias o valores offset (b) de las respectivas capas. Para ello se recurre al entrenamiento de la red. El método de entrenamiento seleccionado fue el algoritmo Levenberg-Marquardt (trainlm), este es un método basado en mínimos cuadrados iterativos (Lampton, 1996), donde el rendimiento de la red se calcula con la raíz del error cuadrático medio (RECM), dado por (3).

$$
\operatorname{RECM}=\sqrt{\frac{\sum\left(\mathrm{y}_{\mathrm{i}}-\mathrm{y}_{\mathrm{i}}^{*}\right)^{2}}{\mathrm{n}}}
$$

Siendo $y_{i}$ el valor del precio de bolsa conocido, $y_{i}^{*}$, el precio predicho por la red y $n$, el número de periodos de tiempo, para este caso 135. 


\section{PRUEBAS Y RESULTADOS}

Se recopiló la información de las entradas y la salida del modelo. En este se compararon diferentes modelos variando el número de neuronas y de retardos. De los 135 datos, el $70 \%$ se utilizó para el entrenamiento, el $15 \%$ para la validación y $15 \%$ para la prueba.

Para la selección del modelo se tuvo en cuenta el mejor desempeño de la red dado por la raíz del error cuadrático medio (RECM) y por el coeficiente de determinación (R). Este coeficiente está dado por (4).

$$
\mathrm{R}=1-\frac{\sum_{\mathrm{i}=1}^{\mathrm{n}}\left(\mathrm{y}_{\mathrm{i}}-\mathrm{y}_{\mathrm{i}}^{*}\right)^{2}}{\sum_{\mathrm{i}=1}^{\mathrm{n}}\left(\mathrm{y}_{\mathrm{i}}-\mathrm{ym}\right)^{2}}
$$

Donde, ym es el promedio de los datos de entrada del precio de bolsa promedio mensual. $\mathrm{y}_{\mathrm{i}}^{*}$, el precio predicho por la red. Y yi el valor del precio de bolsa conocido. En laTabla se presentan los resultados obtenidos cambiando el número de neuronas y de retardos.

Tabla 1: Comparación de resultados con diferentes neuronas y retrasos

\begin{tabular}{|c|c|c|c|c|c|c|c|}
\hline \multirow{2}{*}{$\begin{array}{c}\text { Número de } \\
\text { neuronas }\end{array}$} & \multirow{2}{*}{$\begin{array}{c}\text { Número de } \\
\text { retardos }\end{array}$} & \multicolumn{2}{|c|}{ Entrenamiento } & \multicolumn{2}{c|}{ Validación } & \multicolumn{2}{c|}{ Prueba } \\
\cline { 3 - 8 } & $R E C M$ & $R$ & $R E C M$ & $R$ & $R E C M$ & $R$ \\
\hline 7 & 2 & 16.97 & 0.93 & 17.16 & 0.92 & 19.88 & 0.87 \\
\hline 7 & 3 & 17.21 & 0.92 & 20.07 & 0.91 & 27.63 & 0.80 \\
\hline 8 & 2 & 15.85 & 0.93 & 18.29 & 0.92 & 26.14 & 0.89 \\
\hline 8 & 3 & 13.60 & 0.96 & 15.17 & 0.93 & 21.98 & 0.82 \\
\hline 9 & 3 & 7.00 & 0.99 & 20.42 & 0.92 & 27.22 & 0.80 \\
\hline 8 & 5 & 16.46 & 0.93 & 24.26 & 0.85 & 20.29 & 0.92 \\
\hline 11 & 4 & 16.72 & 0.94 & 24.47 & 0.87 & 21.90 & 0.87 \\
\hline 12 & 4 & 9.22 & 0.98 & 18.62 & 0.90 & 19.03 & 0.87 \\
\hline
\end{tabular}

Al analizar la raíz del error cuadrático medio (RECM) y el coeficiente de determinación (R) en los modelos evaluados, se observa que la estructura de red neuronal con 12 neuronas y 4 retardos, es la que presenta un mejor desempeño. Con este modelo se obtuvo el pronóstico ilustrado en la

Fig. 6 y en la Fig. 7se presenta el análisis de correlación contodos los datos entre enero de 2003 y marzo de 2014 utilizados por la RNA (entrenamiento, validación y prueba), observándose que la red neuronal, permite reproducir adecuadamente la dinámica del precio de la energía eléctrica, lo cual se puede evidenciar a través del RECM de 12.93 y del coeficiente de determinación de $96 \%$. Este coeficiente indica que del total de datos, el $96 \%$ se explican con el modelo.

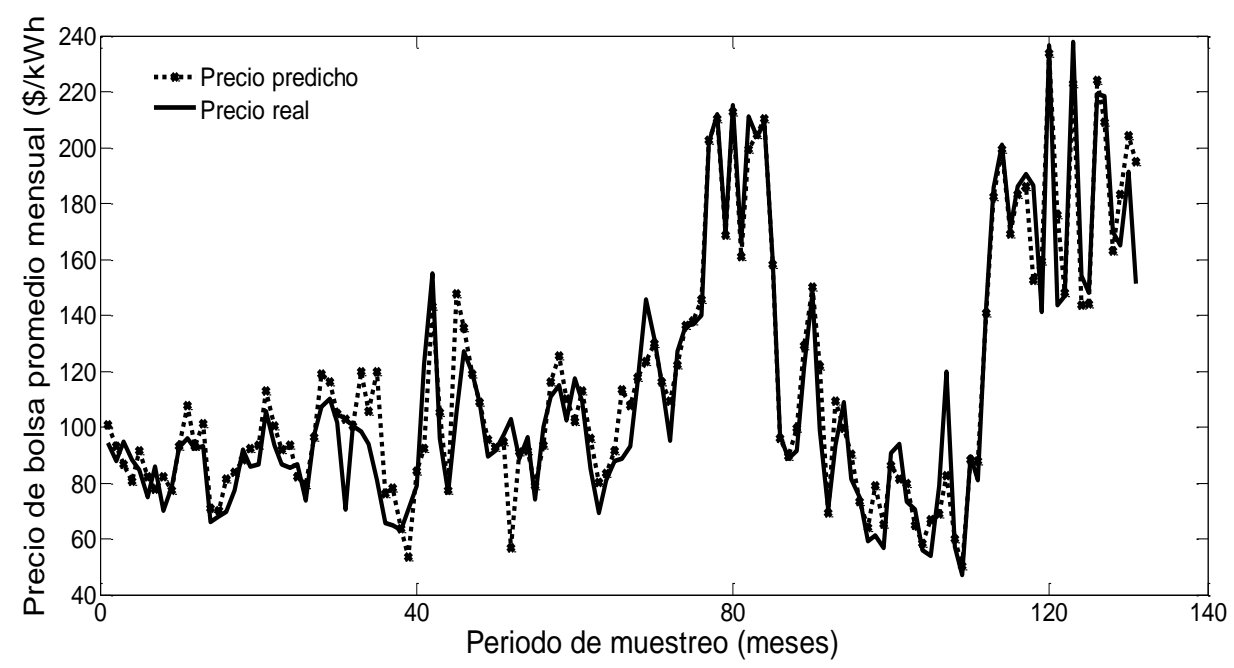

Fig. 6: Pronóstico de la red neuronal dentro de la muestra 


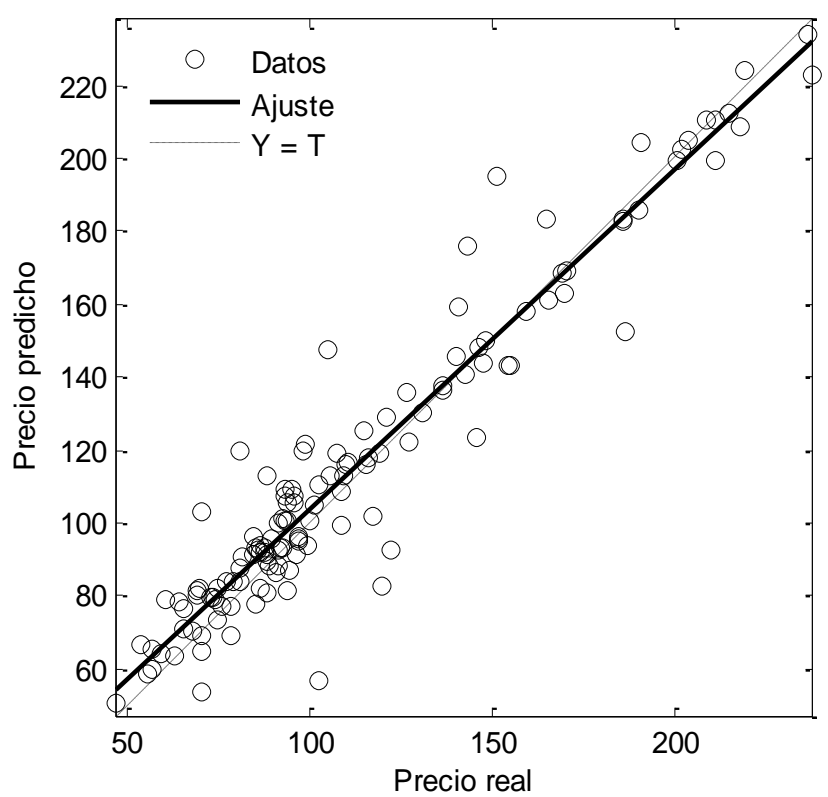

Fig. 7: Coeficiente de correlación obtenidos con la RNA en los diferentes conjuntos

Con el fin de evaluar la capacidad predictiva del modelo, se realizaron pruebas sobre la serie residual dada por (5), para verificar que el error encontrado entre los datos reales ( $\mathrm{y}$ real) y los obtenidos con el modelo (ymodelo) sea un ruido blanco. Esto se puede demostrar a través del análisis de correlación muestral (ACF) y autocorrelación muestral parcial (PACF). En ambos casos, la serie residual es considerada un ruido blanco si las autocorrelaciones se encuentran dentro de las bandas de confianza del $95 \%$, banda determinada por (6). Los resultados del ACF y del PACF son presentados en la Fig. 8, observándose que la serie sí está en el intervalo de confianza, y por tanto es considerada un ruido blanco.

$$
\mathrm{y}_{\mathrm{r}}=\mathrm{y}_{\text {real }}-\mathrm{y}_{\text {modelo }}
$$

Donde, yreal es el precio de bolsa promedio mensual real, y ymodelo es el precio promedio mensual predicho por el modelo.

$$
\left[-\frac{1.96}{\sqrt{T}}, \frac{1.96}{\sqrt{T}}\right]
$$

Donde $T$ es el número de datos de la muestra (131), y por tanto la banda de confianza estará entre [-0.17, $0.17]$. La serie residual está dada por (6).

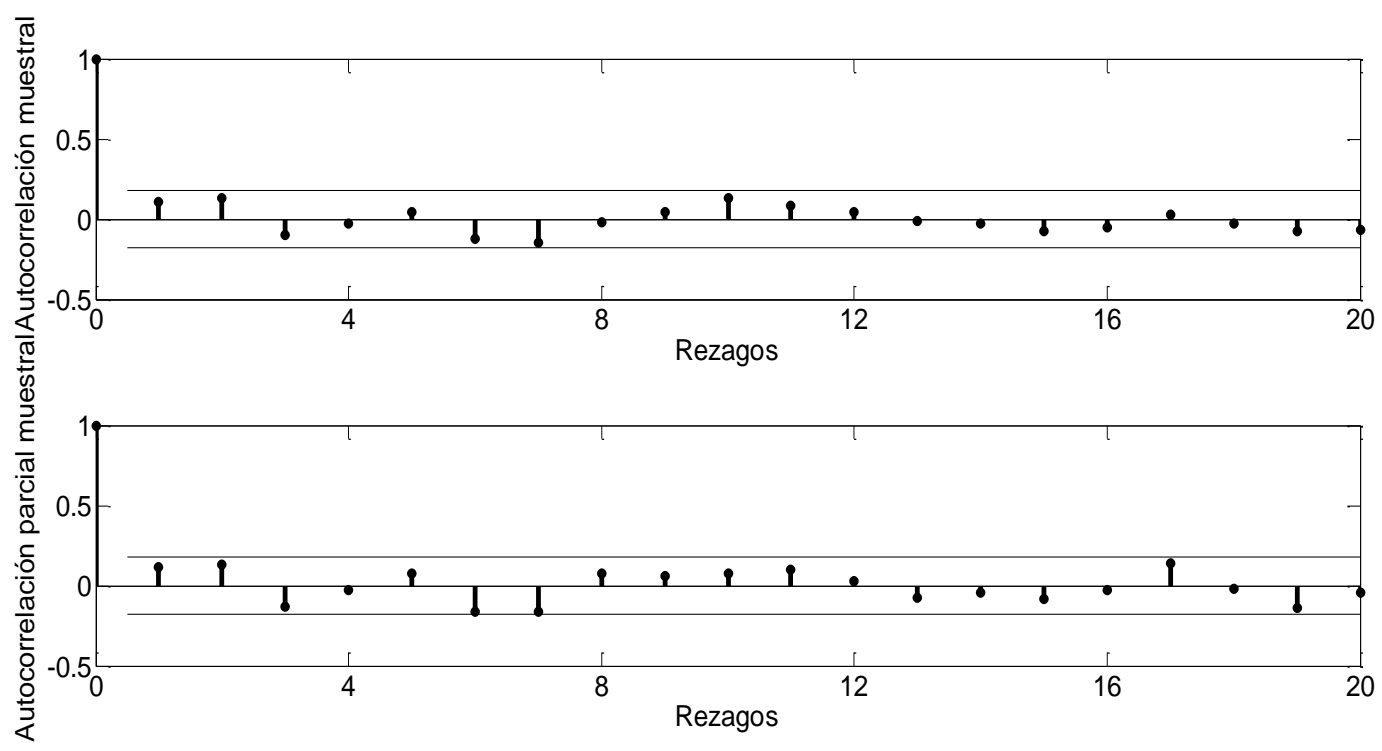

Fig. 8: Autocorrelación muestral y autocorrelación parcial muestral de la serie residual 
También se realizó la prueba de Ljung-Box, prueba estadística determinada por (7).

$$
\mathrm{Q}=\mathrm{T}(\mathrm{T}+2) \sum_{\mathrm{h}=1}^{\mathrm{m}} \frac{\rho(\mathrm{k})^{2}}{\mathrm{~T}-\mathrm{r}}
$$

Donde $\rho(k)$ es la autocorrelación de los residuos en el retardo $\mathrm{k}$, T es el tamaño de la muestra y $\mathrm{m}$ el número de rezagos de la autocorrelación.

La hipótesis nula es que no hay autocorrelación. Los resultados se aprecian en la Tabla 2.Se acepta la hipótesis de que no hay correlación por lo que los residuos son ruido blanco. Por las anteriores pruebas a la serie residual se puede concluir que el modelo capturó bien la información.

Con la finalidad de validar la efectividad del modelo, este fue probado con datos entre abril de 2014 y febrero de 2015. El pronóstico con datos dentro y fuera de la muestra se aprecia en la

Fig. 9 y la regresión para todos los datos se presenta en la Fig. 10. Con los datos de la muestra y fuera de la muestra se obtuvo un RECM de 27.20 y el análisis de coeficientes de determinación indicó que el $88.5 \%$ de los datos se explican con el modelo.

Tabla 2: Prueba de Ljung-Box

\begin{tabular}{|c|c|}
\hline Número de rezagos & $p$-value \\
\hline 10 & 0.22 \\
\hline 20 & 0.61 \\
\hline
\end{tabular}

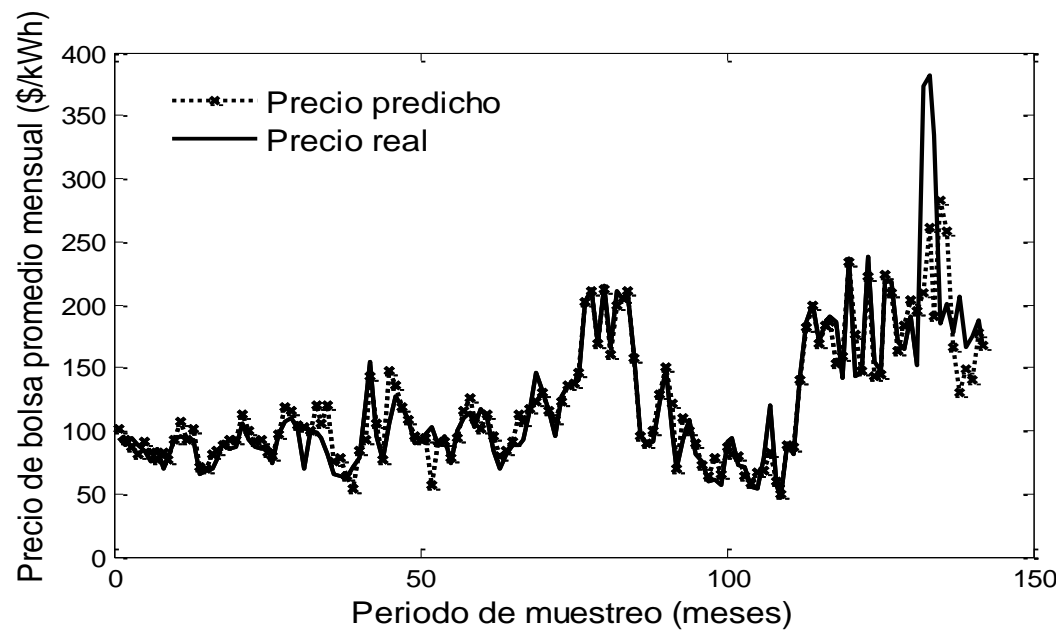

Fig. 9: Pronóstico de la red neuronal dentro de la muestra y con datos fuera de la muestra

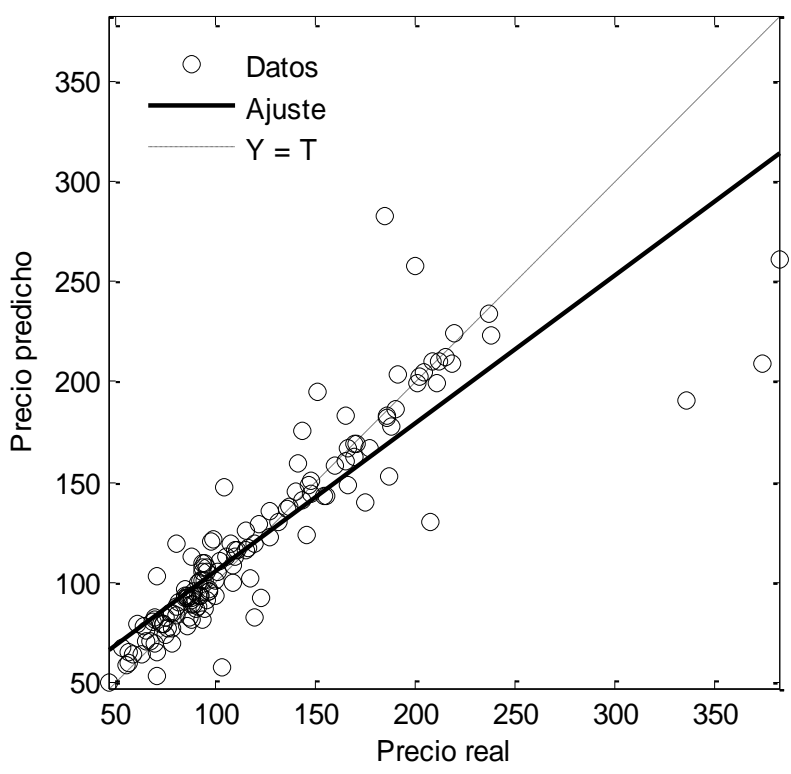

Fig. 10: Coeficiente de determinación $(\mathrm{R})$ para los datos completos dentro y fuera de la muestra 


\section{CONCLUSIONES}

En este trabajo se exploró el problema de predicción de precios en corto plazo para el sistema eléctrico colombiano mediante un modelo basado en redes neuronales artificiales autorregresivas, NARX. El modelo consideró 4 variables exógenas: 3 que dependen de las condiciones hidrologías (la relación entre generación hidráulica-térmica, probabilidad del Fenómeno de El Niño y volumen útil diario energía), y la demanda de energía eléctrica. Adicionalmente se consideraron 4 retardos de la variable de interés.

Los resultados obtenidos con el modelo NARX seleccionado son consistentes con la serie de precios real, lo cual se evidencia a través del alto coeficiente de determinación del $96 \%$ entre los datos reales y los simulados con el modelo, y de una correlación muestral (ACF) y autocorrelación muestral parcial (PACF) del error que se encontró dentro de las bandas de confianza del 95\%. Por esta razón, el modelo de redes neuronales NARX, resulta ser una herramienta apropiada para realizar pronósticos de los precios de la electricidad, ya que con la combinación redes neuronales y series de tiempo, se puede llevar en cuenta la dinámica del precio (no lineal y volátil), encontrar patrones entre las variables de entrada, y tener presente la historia de la variable de interés, condiciones que se adaptan muy bien al paradigma desarrollado en las redes neuronales.

Por otro lado, predecir el valor exacto del precio de bolsa es una tarea compleja y en algunos casos difícil de lograr debido a la gran cantidad de elementos endógenos y exógenos involucrados en su formación. Sin embargo, el modelo propuesto en este artículo puede proporcionar una buena aproximación en el corto plazo, debido a la incorporación de variables que dependen de las condiciones hidrológicas, incluyendo el Fenómeno del Niño que podría ser considerado como una variable especulativa, y de la consideración de la historia del precio.

\section{REFERENCIAS}

Agudelo, L., Velilla, E. y López-Lezama, J.M., Estimación de la cargabilidad de transformadores de potencia utilizando una red neuronal artificial, Información Tecnológica, 25(2), 15-24, (2014)

Amjady, N., Day-Ahead Price Forecasting of Electricity Markets by a New Fuzzy Neural Network, IEEE Transactions on Power Systems, 21(2), 87-896, (2006)

Anbazhagan, S. y Kumarappan, N., Day-Ahead Deregulated Electricity Market Price Forecasting Using Recurrent Neural Network, IEEE Systems Journal, 7(4), 866-872, (2013)

Barrientos, J., Rodas, E., Velilla, E., Lopera, M., y Villada, F., Modelo para el pronóstico del precio de la energía eléctrica en Colombia. Lecturas de Economía, 77(2), 91-127, (2012)

Botero, S. y Cano, J.A., Estado del arte en la estimación de los precios de la energía en el mercado spot, Energética, 23-30, (2007)

Botero, S. y Cano, J.A., Análisis de series de tiempo para la predicción de los precios de la energía en la bolsa de Colombia, Cuadernos de Economía, 48, 174-208, (2008)

Catalão, J.P.S., Pousinho, H.M.I. y Mendes, V.M.F., Short-term electricity prices forecasting in a competitive market by a hybrid intelligent approach, Energy Conversion and Management, 52, 1061-1065,(2011)

Conejo, A. J., Plazas, M.A., Espínola, R. y Molina A.B., Day-Ahead Electricity Price Forecasting Using the Wavelet Transform and ARIMA Models, IEEE Transactions on Power Systems, 20(2), 1035-1042, (2005)

CIDET, Fenómeno del Niño no subirá precios de la energía, 10 de marzo de 2015 , http://www.cidet.org.co/fenomeno-del-nino-no-subira-precios-de-la-energia, Colombia (2014)

De la Fuente, M.J. y Calonge, T, Aplicaciones de las redes de neuronas en supervisión, diagnosis y control de procesos, 17-18. Editorial Equinoccio, Caracas, Venezuela (1999)

García, R., Contreras, J., van Akkeren, M. y García, J.B.C., A GARCH Forecasting Model to Predict DayAhead Electricity Prices, IEEE Transactions on Power Systems, 20(2), 867-874, (2005)

Lampton, M., Damping-undamping strategies for the Levenberg-Marquardt nonlinear least-squares method, Center for EUV Astrophysics, University of California, (1996) 
Nogales, F.J., Contreras, J., Conejo, A.J. y Espínola, R., Forecasting Next-Day Electricity Prices by Time Series Models, IEEE Transactions on Power Systems, 17(2), 342-348, (2002)

Areekul,P., A.,Senjyu, T., Toyama, H. y Yona, A., Hybrid ARIMA and Neural Network Model for Short-Term Price Forecasting in Deregulated Market, IEEE Transactions on Power Systems, 25(1), 524-530, (2010)

UPME, Estudio para determinar la vulnerabilidad y las opciones de adaptación del sector energético, http://www1.upme.gov.co/sites/default/files/vulnerabilidad_opciones_adaptacion_sector_energetico_colombi ano_frente_cambio_climatico.pdf, 20 de enero de 2015, Bogotá, D.C. (2013)

Velásquez, J.D., Dyner, I. y Souza, R.C., Modelado del precio spot de la electricidad en Brasil usando una red neuronal autorregresiva, Revista chilena de ingeniería, 16(3), 394-403, (2008)

Velilla E., Valencia J., Jaramillo F., Performance evaluation of two solar photovoltaic technologies under atmospheric exposure using artificial neural network models, Solar Energy, 107, 260-271, (2014)

Villada, F., García, E. y Molina, J.D., Pronóstico del Precio de la Energía Eléctrica usando Redes NeuroDifusas, Información Tecnológica, 22(6), 111-120, (2011)

Weron, R. y Misiorek, A., Forecasting spot electricity prices with time series models, The European Electricity Market EEM-05, 133-141, Lodz, Polonia, mayo 10-12 (2005) 